\title{
Sudden severe visual loss associated with peripapillary burns during panretinal argon photocoagulation
}

\author{
MANO SWARTZ, DAVID J. APPLE, AND DONNELL CREEL
}

From the Department of Ophthalmology, University of Utah School of Medicine, 50 North Medical Drive, Salt Lake City, Utah 84132, and the Veterans Administration Hospital, 500 Foothill Boulevard, Salt Lake City, Utah 84113, USA

SUMMARY A 31-year-old woman lost central visual acuity in her left eye during scatter argon laser photocoagulation when her ophthalmologist treated the temporal peripapillary region. There was no evidence for a vascular explanation. Previous experimental work showed that the lateral spread of thermal energy into the optic nerve parenchyma from the peripapillary pigment epithelium and choroid can destroy adjacent nerve fibres. There is a risk of thermal optic neuropathy caused by peripapillary photocoagulation.

In 1976 Apple and coauthors ${ }^{1}$ demonstrated in monkey and human eyes that peripapillary argon photocoagulation caused not only overlying retinal burns but also lesions within the adjacent optic nerve parenchyma. Recently, a 31-year-old woman consulted us because she had experienced sudden, severe loss of vision during scatter argon laser photocoagulation for proliferative diabetic retinopathy. Photocoagulation lesions were noted to have been placed on the temporal peripapillary retina and were correlated with arcuate scotomas involving fixation. We believe that she had thermal optic neuropathy due to heat transfer into the optic nerve at sites of macular nerve bundle entry.

\section{Case history}

A 31-year-old woman with type I diabetes of 25 years' duration consulted us to learn why she had suddenly lost her central vision during a session of scatter argon laser photocoagulation performed elsewhere by a retinal specialist. She gave a history of proliferative diabetic retinopathy in both eyes; she had received 8 sessions of photocoagulation in the right eye and 3 sessions in the left eye. It was during the last session in the left eye that she lost her central vision. She did not have a retrobulbar anaesthetic.

Correspondence to Mano Swartz, MD, Department of Ophthalmology, University of Utah Medical Center, 50 North Medical Drive, Salt Lake City, Utah 84132, USA.
On examination her vision was correctable to $6 / 12$ in the right eye and 6/60 in the left eye. There was a left afferent pupillary defect. With the Hardy-RandRitter colour vision test she missed 5 out of 10 plates in the right eye and 6 out of 10 plates in the left eye. Anterior segment biomicroscopy was unremarkable, and her intraocular pressure was $16 \mathrm{mmHg}$ in both eyes. Funduscopic examination of the right eye disclosed involuted proliferative diabetic retinopathy with fibrous membranes along the superotemporal, inferotemporal, and superonasal vascular arcades. A few perfused neovascular vessels remained, and old vitreous blood had gravitated inferiorly. There was a panretinal photocoagulation pattern of approximately 3,200 lesions. There was evidence of substantial treatment within the temporal arcades up to the foveal region (Fig. 1). There were tractional macular folds but no oedema.

Funduscopic examination of the left eye revealed no vitreous haemorrhage and, despite a complete panretinal photocoagulation, 2 patches of perfused retinal neovascularisation along the superotemporal arcade. There were several photocoagulation lesions adjacent to the temporal border of the optic nerve (Fig. 2). This appeared to explain the loss of vision. There was no macular oedema, but both retinas had apparent atrophy of the nerve fibre layer with redfree viewing.

Her visual fields showed generalised construction in the right eye and, in the left eye, a central scotoma with a step at the horizontal meridian (Fig. 3). 


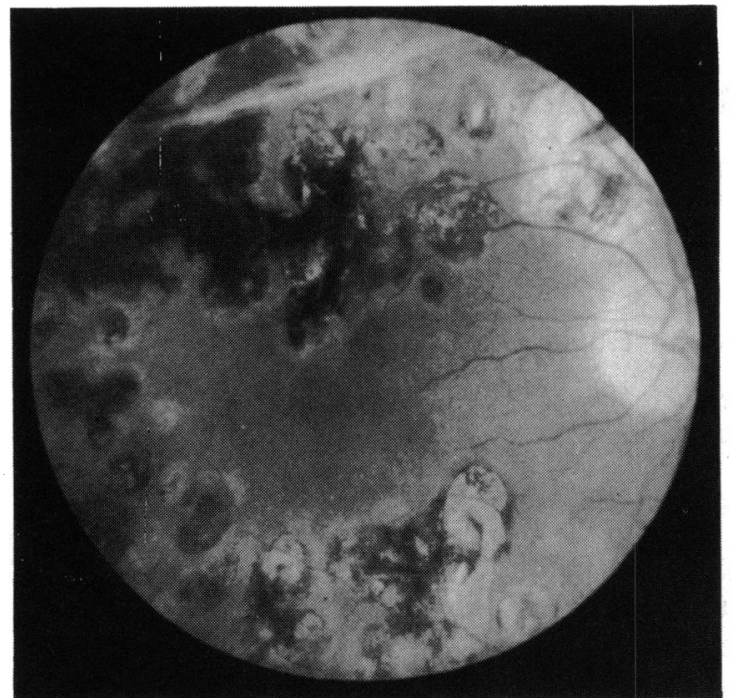

Fig. 1 Scatter photocoagulation in the right eye extends well within the temporal vascular arcades. Some of the lesions are contiguous and heavily pigmented.

The visual evoked potential (VEP), with $50^{\circ}$ arc checks reversed at a rate of $2 / \mathrm{s}$, was abnormal in both eyes. The first positive wave was at $132 \mu \mathrm{s}$ in the right eye and $122 \mu$ s in the left eye (normal is less than 115 $\mu \mathrm{s})$. The amplitudes were normal.

Fluorescein angiography revealed moderately good retinal and optic nerve perfusion without late leakage (Fig. 4).

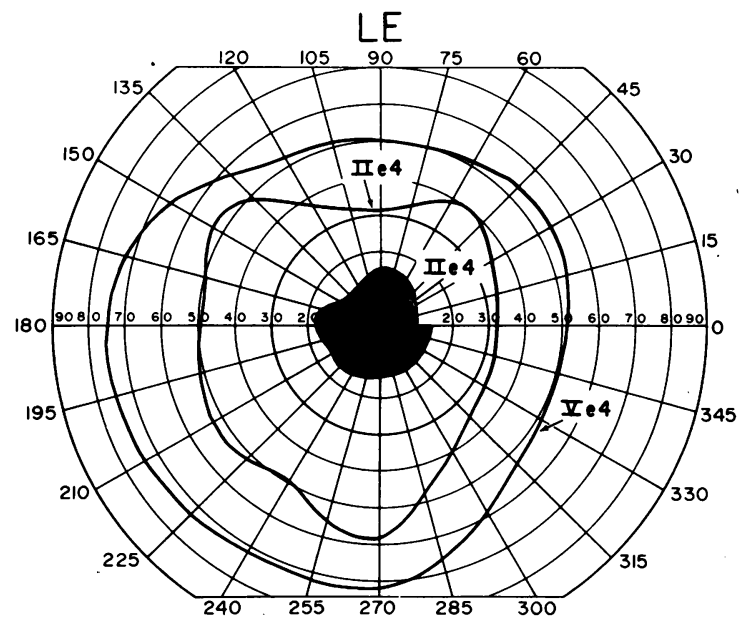

Fig. 3 A large central scotoma correlates with the temporal peripapillary laser lesions and the history of a sudden decrease in vision to 6/60. The step at the horizontal meridian suggests confluent arcuate scotomas.

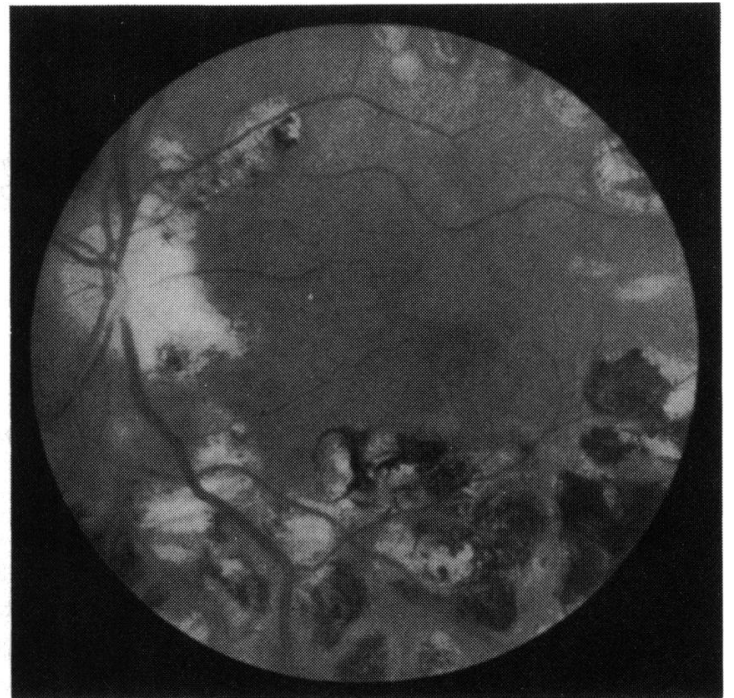

Fig. 2 Several of the photocoagulation lesions in the left eye are located in the temporal peripapillary retina. Heat transfer into the adjacent optic nerve probably was destructive to entering macular nerve fibres.

\section{Discussion}

Zweng and coauthors were the first to point out the dangers of damage to the nerve fibre layer (NFL) and optic nerve when treating on the disc, adjacent to the disc, or in an area of retinal haemorrhage. ${ }^{2}$ The first detailed histopathological and ultrastructural analysis of the argon laser lesion in human retinal and

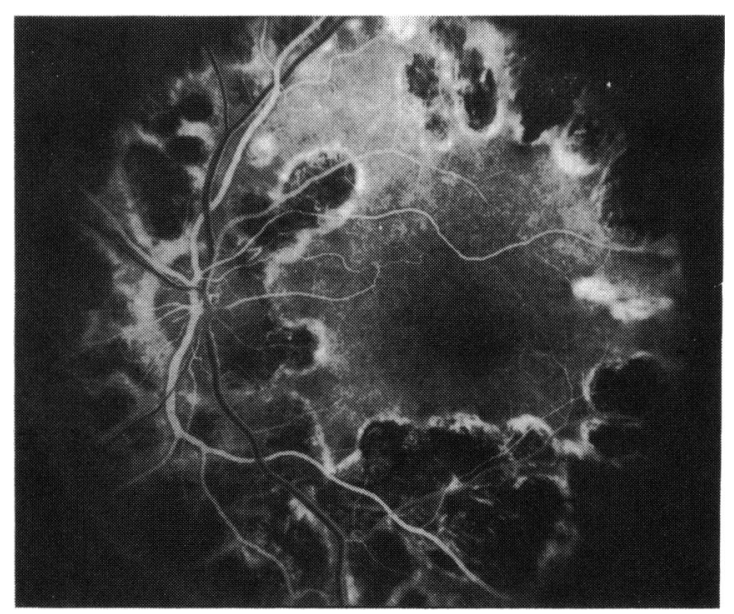

Fig. 4 Fluorescein angiography shows moderately good optic nerve and retinal perfusion. The decrease in vision does not appear to be explained by an ischaemic injury. 
choroidal vasculatures was performed by Apple and coauthors in $1973 .{ }^{3}$ They emphasised the potential for significant damage to the nerve fibre layer from clinically applied retinal burns from an argon laser.

In 1976 Apple and coauthors' ${ }^{1}$ studied 8 monkeys and 5 humans who were subjected to argon laser photocoagulation on the optic nerve head. Energies greater than $400 \mathrm{~mW}, 0 \cdot 2 \mathrm{~s}$, and $100 \mu \mathrm{m}$ spot size, consistently caused neuroparenchymal damage despite the absence of a heat-generating pigment epithelium. These lesions were small, focal areas of degeneration and were therefore considered relatively safe except for the occasional involvement of critical foveal fibres. The threshold for toxicity following peripapiilary photocoagulation was found to be lower because the thermal transformation at the level of the pigment epithelium radiated heat into the adjacent optic nerve fibres. It was also emphasised in this study that normal capillaries within the optic nerve were seldom destroyed or occluded, even after high-energy densities. This led to the conclusion that nerve fibre damage following peri- or epipapillary treatment can be caused by direct thermal destruction of the nerve fibres. Clinically, notable visual field loss following direct epipapillary treatment was reported by Goldberg and Herbst. ${ }^{4}$

The lesions at the superotemporal and inferotemporal rims of the optic nerve head seen in the present case correlate directly with the lesions reported histopathologically by Apple and coauthors. ${ }^{1}$ When the pigment epithelium absorbs incoming argon laser energy, heat is emitted in all directions. When the peripapillary region is treated, there is thermal damage to the adjacent optic nerve as well as to the retina and choroid (Fig. 5). The histopathological correlate of this neuronal degeneration includes acute cystic necrosis and degeneration of the nerve fibre axons as they pass round the border of the rim of the optic nerve, as well as axonal swellings of the treated area, forming massive cytoid bodies. ${ }^{1}$ Laserinduced cytoid bodies have also been observed recently by Thomas, Apple, and Swartz following argon and krypton laser treatment of retinal vessels (manuscript in preparation).

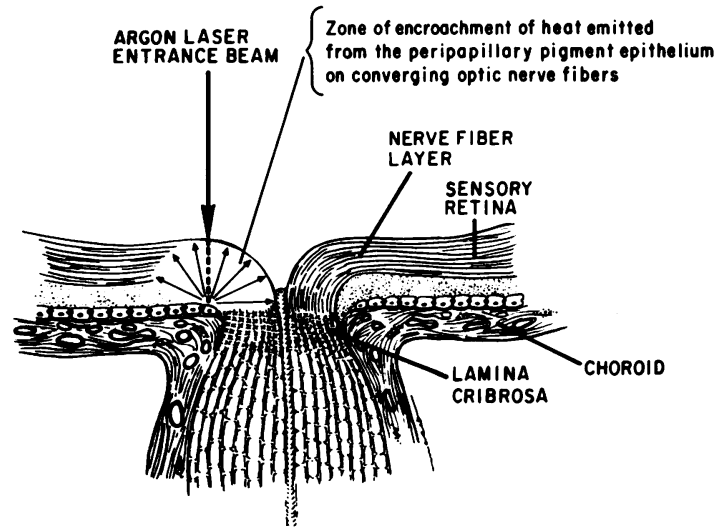

Fig. 5 Thermal energy spreads in all directions from the site of argon laser absorption by the pigment epithelium. The arrows show that nerve fibre layer damage can occur both in the overlying retina and in the adjacent optic nerve.

The complication of thermal optic neuropathy secondary to peripapillary photocoagulation has not been widely discussed recently. In an era in which photocoagulation has been shown to be of benefit for proliferative diabetic retinopathy and subretinal neovascularisation secondary to senile macular degeneration (SMD), the ophthalmologist giving treatment needs to allow for lateral thermal extension when treating in the region of the optic nerve. We believe that this is a potential complication with lasers of any wavelengths that are absorbed by the retinal pigment epithelium and choroid.

\section{References}

1 Apple DJ, Wyhinny GJ, Goldberg MF, Polley EH. Experimental argon laser photocoagulation. II. Effects on the optic disc. Arch Ophthalmol 1976; 94: 296-304.

2 Zweng HC, Little HL, Vassiliadis A. Argon laser photocoagulation. St Louis: Mosby, 1977: 292-307.

3 Apple DJ, Goldberg MF, Wyhinny G. Histopathology and ultrastructures of the argon laser lesion in human retinal and choroidal vasculatures. Am J Ophthalmol 1973; 75: 595.

4 Goldberg MF, Herbst RW. Acute complications of argon laser photocoagulation: epipapillary and peripapillary neovascularization. Arch Ophthalmol 1973; 89: 311-8. 